\title{
AUTO-ASSOCIAÇÃO DO DODECILSULFATO DE SÓDIO (SDS) COM O POLÍMERO HIDROFOBICAMENTE MODIFICADO ETIL(HIDROXIETIL) CELULOSE (EHEC)
}

\author{
Samuel de Medeiros Modolon*, Alexandre Gonçalves Dal Bó, Arlindo Cristiano Felippe, Edson Minatti e Dino Zanette \\ Departamento de Química, Universidade Federal de Santa Catarina, 88040-900 Florianópolis - SC, Brasil
}

Recebido em 7/8/08; aceito em 24/4/09; publicado na web em 22/9/09

\begin{abstract}
SELF-ASSOCIATION OF SODIUM DODECYL SULFATE (SDS) WITH THE HYDROPHOBICALLY MODIFIED POLYMER ETHYL (HYDROXYETHYL) CELLULOSE (EHEC). Mixtures of ethyl(hydroxyethyl)cellulose (EHEC) and Sodium Dodecyl Sulfate (SDS) were investigated using surface tension, conductivity and viscosity measurements in aqueous solutions. The parameters of the surfactant to polymer association processes such as the critical aggregation concentration (cac) and saturation of the polymer by SDS (psp) were determined from the plots of surface tension and specific conductivity versus surfactant concentration. Through the final results we see that there was no specific link of polymer with the surfactant, implying therefore a phenomenon of only cooperative association.
\end{abstract}

Keywords: ethyl(hydroxyethyl)cellulose (EHEC); polymer-surfactant interaction; Sodium Dodecyl Sulfate (SDS).

\section{INTRODUÇÃO}

Nas últimas décadas, a interação entre surfactantes aniônicos e polímeros neutros hidrossolúveis tem sido tema de intensa investigação com finalidade de identificar em solução as propriedades físicas acompanhadas de possíveis mecanismos que interpretem a associação dos surfactantes ao polímero. ${ }^{1-8} \mathrm{~A}$ associação é vista como sendo um fenômeno induzido pelo polímero e inicia em concentrações menores do que quando na sua ausência e o resultado é a formação de complexos termodinamicamente estáveis, com propriedades físico-químicas diferentes daquelas observadas em soluções micelares. ${ }^{9-13}$

Alguns questionamentos são feitos com relação à natureza da estabilidade da interação entre o surfactante e o polímero. A mais "popular" interpretação refere-se ao caráter hidrofóbico, tanto do surfactante como de seções da cadeia polimérica. Sabe-se, no entanto, que somente hidrofobicidade não abrange todos os casos, embora a literatura tenha, recentemente, relatado diferentes modificações estruturais para deixar surfactantes e polímeros com caráter cada vez mais hidrofóbico e, assim, explorar as consequências. ${ }^{3}$

A partir de um grande número de polímeros não iônicos já investigado, sem dúvida, a celulose hidrofobicamente modificada, etil(hidroxietil) celulose (EHEC), é a mais relevante e estudada. EHEC consiste de uma celulose modificada através da introdução de substituintes hidroxietil e etil às correntes da celulose principalmente para aumentar a solubilidade em água. Assim, EHEC pode ser classificado como um polímero, não iônico, anfifílico e solúvel em água. ${ }^{14}$ Essas mudanças químicas resultam em alterações significativas nas propriedades dele, além de conferir maior resistência química, resistência a ataques enzimáticos e promoverem efeitos sinergísticos sobre propriedades reológicas das soluções como em formulações de cosméticos e em tintas à base de água. ${ }^{1-3,15}$

A interação da EHEC com surfactantes aniônicos como o dodecilsulfato de sódio (SDS) tem sido extensivamente pesquisada e os resultados indicam que é governada por forças intrínsecas entre as cadeias e segmentos, agregação entre as cadeias ou por forças eletrostáticas. ${ }^{3,13,16-20} \mathrm{O}$ modelo estrutural dos complexos EHEC-SDS sugerido está fundamentado em medidas de viscosidade e outras propriedades que já foram medidas em função das concentrações do polímero e do SDS em várias temperaturas. ${ }^{19,21}$

\footnotetext{
*e-mail: samuel.modolon@hotmail.com
}

A interpretação estrutural da formação e modificação dos complexos EHEC-SDS induzidas pelos efeitos das concentrações de polímero e do surfactante é assim descrita: (i) Na ausência de surfactante - os domínios hidrofóbicos da cadeia polimérica são responsáveis por formar grupos associados entre as cadeias formando microdomínios hidrofóbicos. Estas associações podem acontecer intramolecularmente e, neste caso, o efeito sobre as propriedades da solução é o da diminuição da viscosidade. Quando a associação é entre moléculas forma em solução uma rede "mais rígida" que aumenta com o aumento da concentração de EHEC. Este último fato justifica o aumento da viscosidade em baixas concentrações de SDS ; ${ }^{19,21}$ (ii) adição de SDS - a interação inicia numa concentração de agregação crítica (cac), comprovada pelas alterações em propriedades já analisadas como diminuição da viscosidade,,$^{1,19,21-23}$ condutividade elétrica $^{2,6,19,23}$ e tensão superficial ${ }^{9,24,25}$. Acima da $c a c$ a tendência é dos agregados micelares no complexo estabelecerem-se nas regiões mais hidrofóbicas, com o aumento da concentração de EHEC. ${ }^{21} \mathrm{~A}$ agregação de polímero com o surfactante termina em uma determinado ponto, (psp), ponto de saturação do polímero.

Acima do $p s p$, as ligações cruzadas começam a romper principalmente por efeito de repulsão eletrostática originada pelo aumento dos agregados micelares na estrutura do complexo. Nesta etapa, aparecem micelas regulares de SDS em equilíbrio com o complexo EHEC-SDS.

Com isso, neste trabalho monitoramos e interpretamos a variação dos parâmetros como cac e psp, indicados por perfis de condutividade elétrica e de tensão superficial versus a concentração de SDS, causada por mudanças da concentração de EHEC em solução aquosa, como uma sustentação para explicar o comportamento dos complexos formados entre EHEC e SDS.

A vantagem de utilizar as técnicas clássicas de tensão superficial e condutividade elétrica reside no fato que elas já foram largamente utilizadas para outros sistemas compostos de misturas de polímeros neutros e surfactantes aniônicos. Assim, as formas e características dos perfis serão utilizadas para visualizar a capacidade de interação do SDS com a celulose. A técnica de condutividade elétrica é extremamente sensível às variações do conteúdo iônico da solução acompanhada de mudanças estruturais, enquanto a tensão superficial, embora uma medida físico-química da interface ar/água, a variação está diretamente em equilíbrio com o interior da solução. Desta forma, embora observem diferentes propriedades em distintas regiões da solução, elas complementam-se nas conclusões. 
Portanto, neste trabalho, aplicamos as técnicas acima relacionadas e justificadas para caracterizar o efeito da concentração do polímero nos parâmetros de ligação, determinar uma correlação da ligação máxima de SDS em função da concentração de EHEC e, finalmente, redefinir o processo de ligação como um processo fortemente cooperativo.

\section{PARTE EXPERIMENTAL}

A etil(hidroxietil) celulose (EHEC), procedente da Bermocoll E 230FQ lot $n^{\circ} 14770$, foi doada pela Akzo Nobel, Surface Chemistry AB, Suécia. A amostra apresenta um grau de substituição de grupos etil $\mathrm{DS}_{\text {etil }}$ de 0,9-1,0 por unidade de anidroglucose e, de uma substituição molar de grupos óxido de etileno, de $\mathrm{MS}_{\mathrm{EO}}$ 1,9-2,2, por unidade de anidroglucose. A massa molar da EHEC, determinada por espalhamento de luz é de $330.000 \mathrm{~g} / \mathrm{mol}$. O polímero tem um grau de polidispersidade $\left(\mathrm{M}_{\mathrm{w}} / \mathrm{M}_{\mathrm{n}}\right)$ abaixo de 2 . O dodecilsulfato de sódio (SDS), com pureza de 99\%, foi obtido da Sigma. Os parâmetros de micelização do SDS foram determinados na ausência e em presença de diferentes concentrações de EHEC.

As soluções estoques de EHEC eram preparadas dissolvendo-se o polímero em água deionizada, sob agitação suave, durante 9-12 h em $(\% \mathrm{~m} / \mathrm{v})$ e, posteriormente, dialisada em membranas de $10.000 \mathrm{~g} / \mathrm{mol}$ durante 5-7 dias em água deionizada, com troca da água duas vezes ao dia. Após, as soluções prontas eram centrifugadas a 4500 rpm durante 25-30 min e filtradas com filtros 0,45 $\mu \mathrm{m}$ (Sartorius). Esta solução era usada para preparar as soluções de SDS. Esta técnica permitiu, durante as titulações condutivimétricas, manter a concentração de EHEC constante e, nas medidas de tensão superficial, soluções livres de partículas em suspensão.

As soluções estoques do tampão foram preparadas a partir de água deionizada Milli-Q e ácido bórico (Vetec). O pH das soluções era ajustado para 9,20 utilizando uma solução de $\mathrm{NaOH}$ padrão.

\section{Método viscosimétrico}

Nas medidas de viscosidade para determinação do regime de concentração da etil(hidroxietil) celulose, $c^{*}$, utilizou-se um viscosímetro com leitor automático de viscosidade da Shott AVS 350 acoplado a um capilar Cannon-Fenske $n^{0} 75$ termostatizado a $25,0{ }^{\circ} \mathrm{C}$ por um banho de água da Schott CT 52. As viscosidades relativas foram obtidas a partir da Equação 1

$\left(\eta / \eta_{\mathrm{o}}=t / t_{\mathrm{o}}\right)$

onde $t$ corresponde ao tempo de escoamento da amostra e $t_{o}$ da solução aquosa.

\section{Tensão superficial}

As medidas de tensão superficial foram feitas utilizando um tensiômetro da Kruss GMBH, modelo K 8, com escala de divisão de $0,1 \mathrm{mN} / \mathrm{m}$, equipado com um anel de Pt-Ir-20 e frasco termostatizado para conter a amostra. A temperatura foi mantida a $25,0 \pm 0,1{ }^{\circ} \mathrm{C}$ através de um termostato.

\section{Condutividade}

As medidas de condutividade específicas foram realizadas utilizando um conditivímetro modelo 170 da Ati-Orion, com uma bureta semi-automática da Metrohm Herisau tipo Multi-Bürette, modelo E-485 com capacidade para $20 \mathrm{~mL}$ e uma cela de diluição. A temperatura foi mantida a $25,0 \pm 0,1^{\circ} \mathrm{C}$ através de um termostato.

\section{RESULTADOS E DISCUSSÃO}

\section{Determinação do regime de concentração da etil(hidroxietil) celulose}

O conhecimento do regime de concentração, adotado na investigação de uma solução polimérica, é tão importante quanto o conhecimento da concentração, tendo em vista que, na passagem de um regime para outro, ocorrem mudanças drásticas nas propriedades da solução. ${ }^{3}$

A Figura 1 mostra um perfil de viscosidade reduzida em função da concentração de EHEC nas temperaturas de 20 e $25,0 \pm 0,1^{\circ} \mathrm{C}$. Os resultados mostram que a viscosidade varia linearmente em função da concentração.

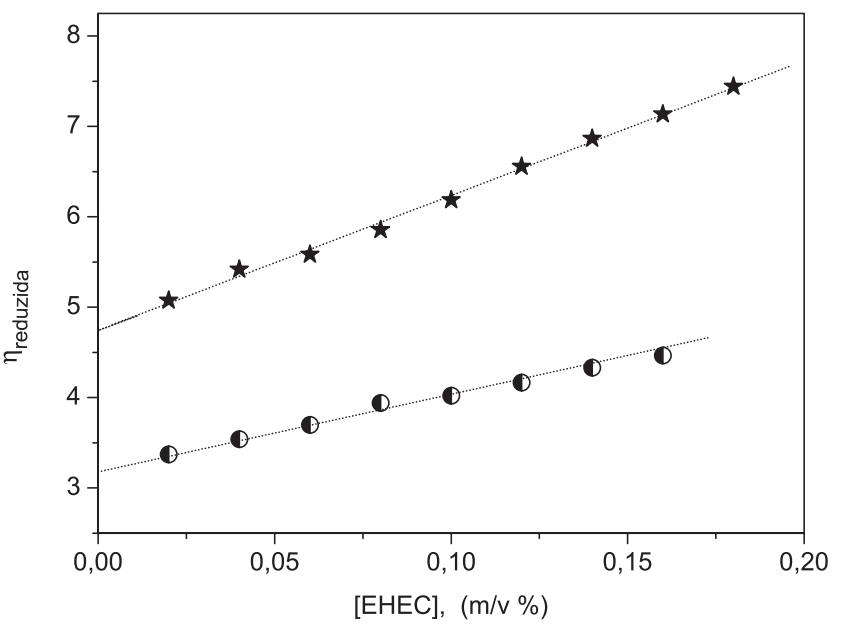

Figura 1. Perfil de viscosidade reduzida em função das concentrações diluídas de EHEC a (x) $20{ }^{\circ} \mathrm{C}$ e (6) $25^{\circ} \mathrm{C}$

Através da extrapolação dos perfis de viscosidade e utilizando a Equação 1, obteve-se o parâmetro c* cujos valores a 25,0 $\pm 0,1$ ${ }^{\circ} \mathrm{C}$, foram de 0,21 e $0,31 \%(\mathrm{~m} / \mathrm{v})$, respectivamente. Estes resultados estão em acordo com aqueles já obtidos por Lindman e Holmberg., ${ }^{4,7}$

Para a EHEC, $\mathrm{c}^{*}$ varia dentro de uma faixa de 0,2 a $0,4 \%(\mathrm{~m} / \mathrm{v})$. Estes valores podem ser alterados em função do aumento da temperatura, da técnica que está sendo utilizada e do grau de substituição na cadeia de celulose dos grupos etil e óxido de etileno.

\section{Tensão superficial}

Historicamente, a tensão superficial é uma das técnicas mais populares para determinação da $c m c$ de tensoativos..$^{27}$ Diferentemente da técnica de condutividade elétrica, que acompanha uma propriedade da solução, a tensão superficial avalia uma propriedade da superfície, na interface ar-água. ${ }^{26,27}$

O polímero EHEC mostra uma forte dependência de adsorção na superfície ar-água em relação ao tempo. O tempo necessário para atingir o equilíbrio entre as formas monoméricas na superfície e na água pode variar desde alguns minutos até horas. Este fato está associado à flexibilidade do polímero e aos grupos substituintes provenientes das modificações químicas que, dependendo da polaridade, apresentam diferentes tendências em adsorverem-se na interface, com mudanças na conformação de suas cadeias. Os grupos etilas têm propriedades hidrofóbicas, portanto, tendem a "escapar da água", enquanto que os grupos oxietilênicos, mais hidrofílicos, por serem mais hidratados tendem ficar no interior da solução. Por medidas de tensão superficial dinâmica na interface ar-líquido, recentemente, Nahringbauer estu- 
dou o tempo de dependência de adsorção do sistema EHEC-SDS. ${ }^{9}$ Por causa deste fato, neste trabalho, em todas as medidas de tensão superficial, as soluções contendo EHEC foram mantidas em repouso de, no mínimo, 60 min antes de serem usadas.

Assim, a Figura 2 mostra o perfil de tensão superficial do SDS em solução tampão, na ausência e em presença de 0,5\% de EHEC. Observa-se que em baixas [SDS], a celulose atua como um tensoativo em soluções aquosas reduzindo a tensão superficial da água-ar.

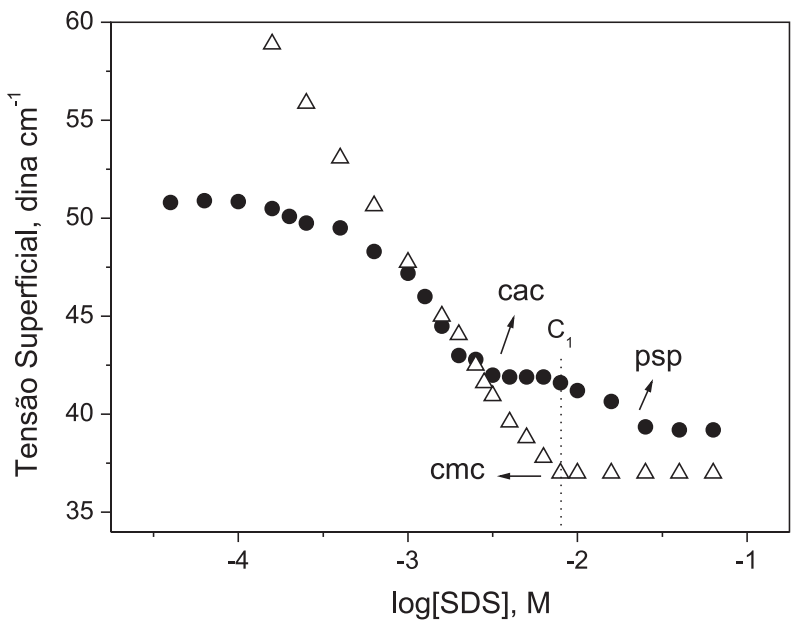

Figura 2. Tensão superficial do SDS na ausência $(\Delta)$ e em presença de $(\bullet)$ $0,5 \%$ EHEC

Em relação à Figura 2, ressaltam-se as seguintes informações: na presença do polímero as diferentes regiões são interpretadas em termos das interações entre o polímero e o surfactante. Inicialmente em baixas concentrações de surfactante, ocorre só atuação dos monômeros dos surfactantes na superfície ar-líquido, enquanto os grupos polares do surfactante ficam na solução aquosa, próximo à superfície minimizando o contato com a água, a atuação do polímero é de minimizar a repulsão entre as cabeças dos surfactantes, ocorrendo assim adsorção constante do surfactante na superfície até ocorrer a total saturação da superfície ar-líquido. ${ }^{28-32}$

O início da associação cooperativa do SDS é alcançado com saturação da superfície, formando complexos EHEC-SDS, é indicado pelo primeiro ponto de inflexão da curva, $c a c$, que ocorre no início do platô em 3,0 $\mathrm{mM}$ de SDS.

O platô, que representa a região da $c a c$ a $\mathrm{C}_{1}$, ocorrendo em 42 dinas $\mathrm{cm}^{-1}$, mostra uma etapa na qual a concentração do SDS na interface ar-água é mantida constante, à medida que se adiciona SDS à solução, cuja extensão do platô depende da concentração do polímero. ${ }^{32-36}$

No final do platô, $\mathrm{C}_{1} \approx 10 \mathrm{mM}$, inicia-se uma nova etapa de concentração monomérica na superfície, a tensão superficial diminui e o processo de associação termina no segundo ponto de descontinuidade, aqui chamado de $p s p .^{26,37}$

O psp, que na Figura 2 ocorre em 26 mM de SDS, representa a concentração na qual ocorre a saturação das cadeias do polímero EHEC por monômeros de SDS. ${ }^{26,38}$

Em concentrações acima do $p s p$, os valores de tensão superficial $\left(\gamma_{2}\right)$ quando na ausência e em presença de EHEC são semelhantes (Tabela 1). Este fato é forte indicação de que, acima do $p s p$, todo SDS adicionado forma micelas livres que estão em equilíbrio com o complexo EHEC-SDS.

$\mathrm{Na}$ ausência do polímero, a $\mathrm{cmc}$ ocorre em 8,2 mM de SDS com valor de tensão superficial de 37,0 dinas $\mathrm{cm}^{-1}$, muito próximo aos valores quando em presença de EHEC (Tabela 1).
Tabela 1. Parâmetros obtidos por tensão superficial para o SDS em diferentes concentrações de EHEC

\begin{tabular}{lccccc}
\hline [EHEC]\% mv & $\mathrm{cmc}, \mathrm{mM}$ & $\mathrm{Cac}, \mathrm{mM}$ & $\mathrm{psp}, \mathrm{mM}$ & $\mathrm{g}_{1}$ & $\mathrm{~g}_{2}$ \\
\hline 0,0 & 8,20 & & & & 37,0 \\
0,05 & & 3,10 & 14,2 & 38,4 & 36,4 \\
0,1 & & 3,15 & 15,1 & 40,0 & 37,0 \\
0,2 & & 3,15 & 17,3 & 40,0 & 37,0 \\
0,25 & 3,20 & 19,0 & 40,5 & 38,0 \\
0,5 & 3,00 & 26,0 & 41,8 & 39,2 \\
0,6 & 3,15 & 28,0 & 40,5 & 38,9 \\
1,0 & 2,90 & 39,0 & 40,0 & 36,9 \\
\hline
\end{tabular}

\section{Condutividade elétrica}

A condutividade é uma propriedade largamente empregada no estudo sobre interação entre polímeros e surfactantes iônicos. Tem sido usada ainda para estimar o grau de ionização $(\alpha)$ de micelas iônicas, assim como para estudar e complementar os processos de associação de micelas iônicas e mistura de surfactantes a polímeros neutros hidrossolúveis. ${ }^{12,39,40} \mathrm{O}$ efeito da hidrofobicidade do contraíon nas interações de surfactantes aniônicos com polímero poli(óxido etilieno) (PEO) e albumina do soro bovino (BSA) foram estudados por Zanette e colaboradores, usando condutividade elétrica e sondas cinéticas. ${ }^{41}$ Zana também usou a técnica para estender a discussão sobre o efeito da hidrofobicidade de contra-íons de diferentes surfactantes do tipo dodecilsulfatos com PEO. ${ }^{42}$

Existem várias técnicas para o cálculo do grau de ionização de micelas iônicas, a mais simples é a condutimétrica, cujo método consiste na razão entre a inclinação da região linear acima $\left(\mathrm{S}_{3}\right)$ e abaixo $\left(\mathrm{S}_{1}\right)$ da $c m c$. A medida e o conhecimento de $\alpha$ são importantes porque estão relacionados com o conteúdo de contra-íons ligados na superfície da micela e com a densidade de carga, portanto, das propriedades físico-químicas da interface micelar.

A partir das medidas de condutividade, foi possível calcular os valores do grau de dissociação, pelo método de $\alpha$ Frahm. ${ }^{43} \mathrm{O}$ método de Frahm assume que as condutividades abaixo e acima da $c m c$ são causadas exclusivamente pelos contra-íons do surfactante.

No cálculo do grau de dissociação pelo método de Frahm, utilizou-se a Equação 2

$\alpha_{1}=\mathrm{S}_{3} / \mathrm{S}_{1}$

O valor de $\alpha_{1} \approx 0,38$ encontrado para solução de SDS na ausência de polímero apresenta boa concordância com os dados da literatura. ${ }^{38,44,45}$

De outro lado, o grau de ionização dos complexos EHEC-SDS $\left(\alpha_{2}\right)$, pode ser estimado a partir da razão $S_{2} / S_{1}$

$\alpha_{2}=S_{2} / S_{1}$

A Figura 3 mostra os perfis de condutividade elétrica versus [SDS] na ausência e em presença de EHEC. Para misturas do polímero EHEC e surfactante, o perfil de condutividade elétrica apresenta dois pontos de inflexão. O primeiro representa o início da associação cooperativa entre o polímero e o surfactante, $c a c$, e o segundo a saturação do polímero por moléculas de surfactante, $p s p$. São características ainda duas regiões lineares abaixo da $c a c$, acima do $p s p$, e uma região entre cac-psp que tem a linearidade dependente do sistema e das circunstancias experimentais, onde pode ser observada em maiores detalhes na Figura 4 e discutidos melhor por Zanette e colaboradores. ${ }^{46}$ 


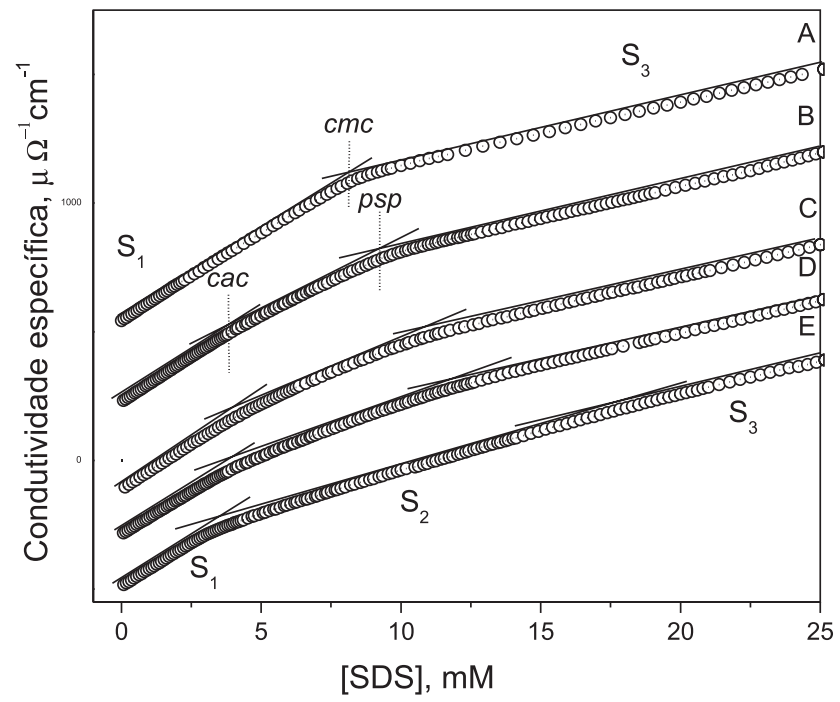

Figura 3. Condutividade específica (em escala relativa) versus [SDS] na ausência (A) de EHEC e em presença de (B) 0,05\%, (C) 0,1\%, (D) 0,2\% e (E) $0,5 \%$ de EHEC

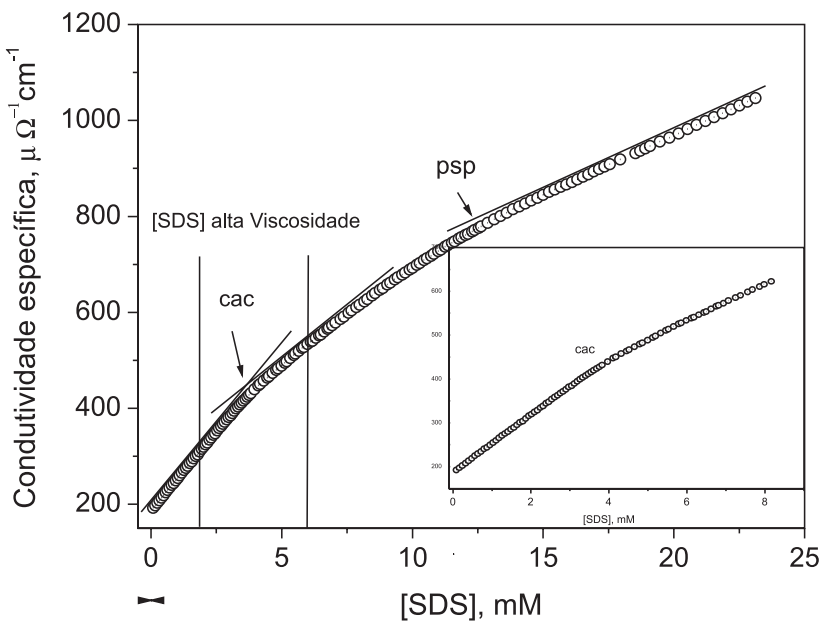

Figura 4. Condutividade específica versus [SDS] em presença de 0,2\% de EHEC

A primeira região linear ocorre em concentrações abaixo da cac. $\mathrm{O}$ coeficiente angular $\mathrm{S}_{1}$, quando mantidas todas as condições experimentais constantes, é numericamente idêntico àquele quando na ausência do polímero. ${ }^{12} \mathrm{~A}$ Tabela 2 lista os valores obtidos para diferentes concentrações de EHEC. Considerando o limite de exatidão da técnica, este fato indica que, abaixo da $c a c$, não deva ocorrer nenhum processo de ligação específica e somente na $c a c$ é que associação cooperativa de agregados micelares inicia a ligação sobre o polímero.
A segunda região linear, localizada acima do ponto de saturação do polímero, é interpretada como sendo uma região onde somente micelas de SDS são formadas em equilíbrio dinâmico com complexos formados até o psp. Uma forte evidência para esta conclusão provém do fato que acima do psp, os coeficientes angulares são idênticos àquele acima da $c m c$ do SDS (Tabela 2).

A região localizada entre os pontos de descontinuidade, cac-psp, é definida como uma etapa de formação de agregados com diferentes características das micelas de SDS, sendo esta uma etapa onde ocorre efeito sinergístico causado pelo processo de ligação e desadsorção. Observa-se que a linearidade na região entre $c a c$ e $p s p$ depende das condições experimentais, o aumento da concentração do polímero induz mudanças significativas nessa região.

Como a adição de um polímero neutro, como no caso o EHEC, numa solução contendo um surfactante, não interfere significativamente na condutividade elétrica da solução, então, mudanças no perfil de condutividade indicam interação entre o polímero e o surfactante.

A Tabela 2 apresenta os valores de $c m c, c a c, p s p$, coeficientes angulares e grau de ionização, obtidos por condutividade elétrica, para diferentes concentrações de EHEC, variando de 0,0 a $1,0 \%$. As razões obtidas pela Equação 2 referem-se ao grau de ionização de micelas regulares, $\alpha_{1}$ e pela Equação 3, referem-se ao grau de ionização de complexos EHEC-SDS, $\alpha_{2}$, respectivamente listados na Tabela 2.

Podemos observar que os valores de $\alpha_{2}$ são maiores que os valores de $\alpha_{1}$, isso indica que complexos EHEC-SDS são mais ionizados que micelas regulares. É interessante observar que o valor de $\alpha_{1}$ para complexos EHEC-SDS é muito semelhante ao valor obtido para SDS puro, então podemos concluir que acima do $p s p$ existem micelas regulares de SDS.

Os resultados obtidos pelos métodos condutométrico e tensiométrico são resumidos no diagrama da Figura 5 variando-se a concentração de EHEC versus SDS. Observam-se, para ambas as técnicas, que o ponto de saturação do polímero, $p s p$, varia linearmente com a concentração de EHEC; este resultado é mais uma evidência da ligação entre o surfactante ao polímero, cujas correlações lineares são definidas pelas Equações 4 e 5, a partir dos gráficos de tensão superficial e de condutividade elétrica, respectivamente, as correlações são análogas às já obtidas por Zanette e colaboradores para o sistema PEO-SDS, EHEC-SDoD. ${ }^{46-48}$

$$
\begin{aligned}
{[\mathrm{SDS}]_{\mathrm{t}} } & =0,026[\mathrm{EHEC}]+0,012(\mathrm{M}) \\
{[\mathrm{SDS}]_{\mathrm{t}} } & =0,017[\mathrm{EHEC}]+0,010(\mathrm{M})
\end{aligned}
$$

O sistema SDS-EHEC exibe correlações lineares bem definidas em relação à cac (Figura 5). Os perfis de cac obtidos por condutividade elétrica e tensão superficial são semelhantes, independentes não mostrando alteração evidenciando ausência de ligação específica da concentração da celulose implicando num fenômeno de associação de forte cooperação

Tabela 2. Parâmetros obtidos por condutividade elétrica especifica para o SDS em diferentes concentrações de EHEC

\begin{tabular}{lcccccccc}
\hline [EHEC] $(\% \mathrm{mv})$ & $\mathrm{cmc}, \mathrm{mM}$ & $\mathrm{cac}, \mathrm{mM}$ & $\mathrm{psp}, \mathrm{mM}$ & $\mathrm{S}_{1}$ & $\mathrm{~S}_{2}$ & $\mathrm{~S}_{3}$ & $\alpha_{1}$ & $\alpha_{2}$ \\
\hline 0 & 8,2 & -- & -- & 66,8 & -- & 25 & 0,38 & 0,36 \\
0,05 & -- & 3,5 & 10,6 & 70 & 52 & 25 & 0,75 \\
0,1 & -- & 3,3 & 11,6 & 69 & 50 & 27 & 0,38 \\
0,15 & -- & 3,3 & 12,2 & 67 & 46 & 27 & 0,40 & 0,69 \\
0,20 & -- & 3,2 & 13 & 65 & 42 & 25 & 0,39 & 0,64 \\
0,50 & -- & 3,3 & 18 & 67 & -- & 26 & 0,39 & -- \\
1,0 & -- & 3,4 & 27 & -- & -- & 25 & -- \\
\hline
\end{tabular}


entre o surfactante SDS e o polímero EHEC, como também já representadas pelo tensoativo SDoD e para mistura de ambos. ${ }^{46}$

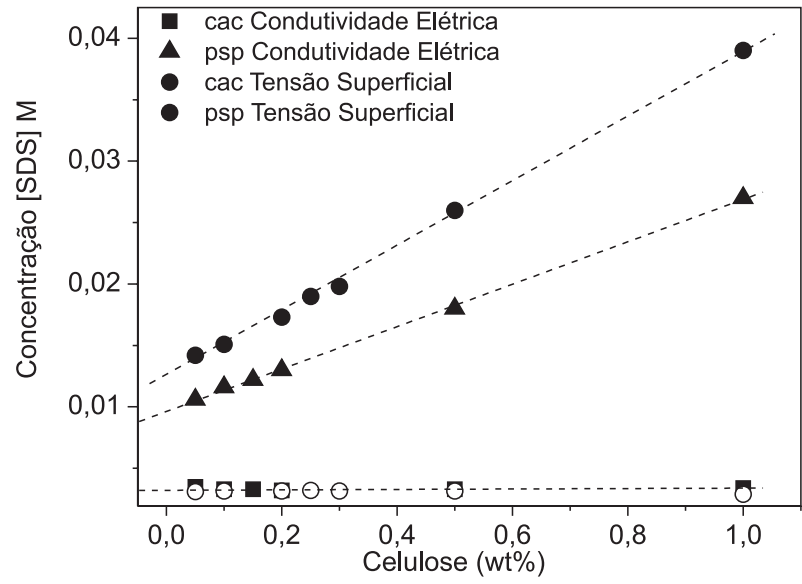

Figura 5. Valores de cac e psp medidos por tensão superficial $(\mathrm{O}) e(\bullet), e$ por condutividade elétrica. (অ) $e(\mathbf{\Delta})$

\section{CONCLUSÕES}

Tanto por condutividade elétrica como por tensão superficial, conclui-se que a concentração de agregação crítica, $c a c$, independe da concentração de EHEC, implicando que o processo de autoassociação do SDS ao EHEC é fortemente cooperativo.

O polímero não demonstra influência no grau de ionização antes da $c a c$ e após psp, tendo influência apenas na faixa que liga os dois pontos, mesmo em regime de concentração diferente da c* ou não, a $c a c$ não mostra alteração evidenciando ausência de ligação específica.

O segundo ponto de descontinuidade das curvas de tensão superficial e condutividade elétrica mostra uma dependência linear da concentração de EHEC, comportamento análogo de sistemas como PEO-SDS e EHEC-SDoD, já obtidas por Zanette e colaboradores. ${ }^{44,46}$

\section{REFERÊNCIAS}

1. Bloor, D. M.; Wan-Yunus, W .M. Z.; Wan-Badhi, w.; Li, Y.; Holzwarth, J. F.; Wyn-Jones, E.; Langmuir 1995, 11, 3395.

2. Zana, R.; Biana-Limbele, W.; Kamenka, N.; Lindman, B.; J. Phys. Chem. 1992, 96, 13

3. Hansson, P.; Lindman, B.; Curr. Opin. Colloid Interface Sci. 1996, 1, 604.

4. Goddard, E. D.; Ananthapadmanabhan, K. P.; Interactions of Surfactants with Polymers and Proteins, $1^{\text {st }}$ ed., CRC Press: Boca Raton, 1993.

5. Shweitzer, B.; Zanette, D.; Itri, R.; J. Colloid Interface Sci. 2004, 277, 285.

6. Kamenka, N.; Burgaud, I.; Zana, R.; Lindman, B.; J. Phys. Chem. 1994, 98, 6785 .

7. Hoff, E.; Nyström, B.; Lindman, B.; Langmuir 2001, 17, 28.

8. Gennes, P. G.; J. Phys. Chem. 1990, 94, 8407.

9. Nahringbauer, I.; Langmuir 1997, 13, 2242.

10. Holmberg, C.; Nilsson, S.; Sundelöf, L. O.; Langmuir 1997, 13, 1392.

11. Karlstrom, G.; Lindman, B. Em Organized Solutions: Surfactants in Science and Technology; Friberg, S. E.; Lindman, B., eds.; Marcel Dekker: New York, 1992, cap. 5.
12. Zanette, D.; Frescura, V.; Colloid Interface Sci. 1999, 213, 379.

13. Nilsson, S.; Holmberg, C.; Sundelöf, L. O.; Colloid Polym Sci. 1995, 273,83 .

14. Karlstrom, G.; Carlsson, A.; Lindman, B.; J. Phys. Chem. 1990, 94, 5005.

15. Mano, E. B.; Introdução a Polímeros, $2^{\mathrm{a}}$ ed., Edgard Blücher: São Paulo, 1985.

16. Galant, C.; Kjoniksen, A. L.; Knudsen, K. D.; Helgesen, G.; Lund, R.; Laukkanen, A.; Tenhu, H.; Nyström, B.; Langmuir 2005, 21, 8010.

17. Zhao, G.; Khin, C. C.; Chen, S. B.; Chen, B. H.; J. Phys. Chem. B 2005 , 109, 14198.

18. Joabsson, F.; Lindman, B.; Prog. Colloid Polym. Sci. 2000, 116, 74.

19. Holmberg, C.; Sundelöf, L. O.; Langmuir 1996, 12, 883.

20. Kjoniksen, A. L.; Knudsen, K. D.; Nyström, B.; Eur. Polym. J. 2005, 41, 1954.

21. Lund, R.; Lauten, R. A.; Nyström, B.; Lindman, B.; Langmuir 2001, 17, 8001.

22. Hoff, E.; Nystrom, B.; Lindman, B.; Langmuir 2001, 17, 28.

23. Holmberg, C.; Nilsson, S.; Singh, S. K.; Sundelöf, L.-O.; J. Phys. Chem. 1992, 96, 871 .

24 Nahringbauer, I.; J. Colloid Interf. Sci. 1995, 176, 318.

25 Djuve, J.; Pugh, R. J.; Sjoblom, J.; Colloids Surf., A 2001, 186, 189.

26 Goddard, E. D.; Colloid Interface Sci. 2002, 256, 228.

27. Menger, F. M.; Galloway, A. L.; Chlebowski, M. E.; Langmuir 2005, 21, 9010.

28. Purcell, I. P.; Thomas, R. K.; Penfold, J.; Howe, A. M.; Colloids Surf., A 1995, 94, 125.

29. Jean, B. L. L. T.; Cabane, B.; Langmuir 1999, 15, 5987.

30. Purcell, I. P.; Thomas, R. K.; Howe, A. M.; Penfold, J.; Langmuir 1998 , 14, 1637.

31. Cabane, B.; J. Phys. Chem. 1997, 81, 1639.

32. Abuin, E.; Leon, A.; Lissi, E.; Varas, J. M.; Colloids Surf., A 1999, 147, 55.

33. Stubenrauch, C.; Klitzing, R. V.; Langevin, D.; Langmuir 2000, 16, 3206 .

34. Schwuger, M. J.; J. Colloid Interface Sci. 1973, 43, 491

35. Myrvold, R.; Hansen, F. K.; J. Colloid Interface Sci. 1998, $207,97$.

36. Zanette, D.; Felippe, A. C.; Schweitzer, B.; Dal-Bó, A.; Lopes, A.; Colloids Surf., A 2006, 279, 87.

37. Jones, M. N.; Colloid Interface Sci. 1967, 23, 36.

38. Rodenas, E.; Sierra, M. L.; Langmuir 1996, 12, 1600.

39. Mukerjee, P.; Mysels, K. J.; Kapauan, P.; J. Phys. Chem. 1967, 71, 4166.

40. Lima, C. F.; Nome, F.; Zanette, D.; Colloid Interface Sci. 1997, 189, 174.

41. Zanette, D.; Lima, C. F.; Ruzza, A. A.; Belarmino, A. T. N.; Santos, S. D. F.; Frescura, V.; Marconi, D. M. O.; Froehner, S. J.; Colloids Surf., A 1999, 147, 89.

42. Benrraou, M.; Bales, B.; Zana, R.; Colloid Interface Sci. 2003, 267, 519.

43. Pires, P. A. R.; Tese de Doutorado, Universidade Federal de São Paulo, Brasil, 2002.

44. Froehner, S. J.; Belarmino, J.; Zanette, D.; Colloids Surf., A 1998, 137, 131.

45. Ruzza, A. A.; Froehner, S. J.; Minatti, E.; Nome, F.; Zanette, D.; J. Phys. Chem. 1994, 98, 12361.

46. Dal Bó, A.; Zanette, D.; Shweitzer, B.; Felippe, A. C.; Lindman, B.; Colloids Surf., A 2005, 256, 171.

47. Minatti, E.; Zanette, D.; Colloids Surf., A 1996, 113, 237.

48. Zanette, D.; Froehner, S. J.; Ruzza, A. A.; Minatti, E.; Colloids Surf., A 1996, $108,91$. 